\title{
THE INTERACTION OF GLUCAGON AND INSULIN ON BLOOD GLUCOSE
}

\author{
By H. ELRICK, C. J. HLAD, JR., Y. ARAI, AND A. SMITH \\ (From the General Medical Research Laboratory and the Radioisotope Service of the \\ Veterans Administration Hospital and the Department of Medicine, University \\ of Colorado Medical School, Denver, Colo.)
}

(Submitted for publication December 27, 1955 ; accepted March 15, 1956)

In previous publications from this laboratory (1, 2) evidence was presented which indicates that glucagon enhances the peripheral utilization of glucose $^{1}$ in normal men and dogs as well as in the depancreatized dog. The purpose of this paper is to report experiments on the combined effect of glucagon and insulin on arterial and venous glucose levels in normal men, and to compare the magnitude of this effect with that observed in subjects given similar amounts of each hormone separately.

\section{METHODS}

Fifty-six men (ages 21 to 47 ) considered normal with respect to carbohydrate metabolism and in good general health and nutritional state were studied. Controlled basal conditions were obtained in the following manner: (a) Subjects were hospitalized and placed on a high carbohydrate $(360 \mathrm{gm}$.), high caloric diet (3500 calories) for at least three days before being studied; (b) a 14hour fast preceded each study; (c) experiments were begun at 8 a.m. following a 30 to 60 -minute period of bed rest in the test bed in a special room; (d) venous blood samples were obtained from an indwelling needle inserted (using local Procaine anesthesia) five minutes before the experiment was begun; (e) every effort was made to keep the subjects motionless, relaxed and comfortable during the experiments.

Ten per cent glucose was infused intravenously at a constant rate $(250$ to $370 \mathrm{mg}$. per min. in different subjects) throughout the test period by means of a Bowman constant infusion pump. The initial 30 to 60 minutes of the infusion period served as a control period, following which glucagon ${ }^{2}$ and/or insulin ${ }^{2}$ was added to the infusion bottle. The glucagon dosage was 0.7 to $1.0 \mathrm{mg}$. administered by constant intravenous infusion over periods

1 Glucose utilization is used to mean the disappearance of blood glucose.

2 Generously supplied by Drs. O. K. Behrens and W. R. Kirtley of Eli Lilly Company. The glucagon (Lot 208-158B-214A) contains 50 per cent of the potency of the crystalline standard and an insulin content of $\mathbf{0 . 0 5}$ to 0.005 units per milligram. The insulin (Lot No. $\mathrm{T}-3206)$ was a preparation free of glucagon. ranging from 60 to 100 minutes. Insulin dosage ranged from 0.054 to 0.128 units per min. by constant intravenous infusion for periods ranging from 65 to $110 \mathrm{~min}$ utes. Mean insulin dosage for the subjects given insulin alone was .076 units per minute, for the subjects given insulin plus glucagon it was .071 units per minute. The dosage of insulin was arrived at by trial and error; the criteria used were that the dose be sufficient to cause a definite drop in blood sugar, but not so large as to result in hypoglycemic values. Data in which arterial blood sugar fell below $65 \mathrm{mg}$. per cent were discarded. In the subjects who received both insulin and glucagon, the insulin infusion was begun 15 to 25 minutes before the glucagon in order to obtain the maximum effect of each hormone (on blood sugar level) at approximately the same time.

Venous and capillary (finger) blood specimens were drawn at five or ten-minute intervals, a total of 30 to 40 samples during each experiment. Venous samples were obtained from the antecubital vein which carries mixed blood from the deep and superficial tissue of the forearm and hand $(3,4)$. Capillary blood contains the same glucose concentration as arterial blood (5). Samples were analyzed for glucose content by the Nelson-Somogyi method (6). Sodium fluoride and immediate refrigeration were used to prevent glycolysis in the samples. Capillary samples were analyzed singly, venous samples in duplicate. In approximately 900 duplicate venous samples the maximum difference between any pair was 10 per cent, and the average was 2 per cent.

The A-V/A data were subjected to a statistical analysis by means of a non-parametric median test $(7)$; i.e., using the medians as a cutting point. The data were placed in a $2 \times 2$ contingency table, and then subjected to a chisquare analysis.

\section{RESULTS}

The data on the 12 subjects who received insulin following an initial control period are given in Table I. The data from one of these experiments are shown in Figure 1. In 11 of the 12 subjects insulin resulted in a fall in arterial and venous blood glucose levels to or below fasting levels in spite of the constant glucose infusion. 
TABLE I

Insulin cases *

\begin{tabular}{|c|c|c|c|c|c|c|c|c|c|c|c|c|c|c|c|c|c|c|}
\hline \multirow{2}{*}{$\begin{array}{c}\text { Patient } \\
W_{t} \\
\left(K_{\boldsymbol{g} .}\right) \\
\end{array}$} & \multirow[b]{2}{*}{ Age } & \multirow{2}{*}{$\begin{array}{c}\text { Glucose } \\
\text { mg. } \\
\text { min. }\end{array}$} & \multirow{2}{*}{$\begin{array}{c}\text { Insulin } \\
\text { units/ } \\
\text { min. }\end{array}$} & & \multicolumn{14}{|c|}{ Time in minutes } \\
\hline & & & & & 0 & 10 & 20 & 30 & 40 & 50 & 60 & 70 & 80 & 90 & 100 & 110 & 120 & 130 \\
\hline $\begin{array}{l}\text { W. Y. } \\
77.8\end{array}$ & 22 & 344 & .128 & $\stackrel{\mathrm{A}}{\mathrm{V} \dagger}$ & $\begin{array}{l}89 \\
87\end{array}$ & $\begin{array}{l}108 \\
105\end{array}$ & $\begin{array}{l}108 \\
105\end{array}$ & $\begin{array}{l}125 \\
123\end{array}$ & $\begin{array}{l}130 \\
128\end{array}$ & $\begin{array}{l}134 \\
132\end{array}$ & $\begin{array}{l}136 \\
135\end{array}$ & $\begin{array}{l}136 \\
130\end{array}$ & $\begin{array}{l}127 \\
121\end{array}$ & $\begin{array}{l}118 \\
111\end{array}$ & $\begin{array}{r}106 \\
99\end{array}$ & $\begin{array}{l}92 \\
85\end{array}$ & & \\
\hline E. J. & 25 & 360 & .088 & $\stackrel{\mathrm{A}}{\mathrm{V}}$ & $\begin{array}{l}86 \\
78\end{array}$ & $\begin{array}{l}105 \\
100\end{array}$ & $\begin{array}{l}114 \\
108\end{array}$ & $\begin{array}{l}120 \\
112\end{array}$ & $\begin{array}{l}124 \\
115\end{array}$ & $\begin{array}{l}127 \\
116\end{array}$ & $\begin{array}{l}129 \\
117\end{array}$ & $\begin{array}{l}130 \\
117\end{array}$ & $\begin{array}{l}128 \\
114\end{array}$ & $\begin{array}{l}117 \\
105\end{array}$ & $\begin{array}{l}99 \\
93\end{array}$ & $\begin{array}{l}85 \\
82\end{array}$ & $\begin{array}{l}77 \\
74\end{array}$ & \\
\hline$\underset{81.6}{\text { R. B. }}$ & 29 & 360 & .113 & $\stackrel{\mathbf{A}}{\mathbf{V}}$ & $\begin{array}{l}97 \\
92\end{array}$ & $\begin{array}{l}109 \\
104\end{array}$ & $\begin{array}{l}120 \\
115\end{array}$ & $\begin{array}{l}129 \\
122\end{array}$ & $\begin{array}{l}134 \\
127\end{array}$ & $\begin{array}{l}138 \\
131\end{array}$ & $\begin{array}{l}140 \\
133\end{array}$ & $\begin{array}{l}135 \\
127\end{array}$ & $\begin{array}{l}122 \\
116\end{array}$ & $\begin{array}{l}106 \\
104\end{array}$ & $\begin{array}{l}90 \\
91\end{array}$ & $\begin{array}{l}76 \\
78\end{array}$ & & \\
\hline C. J. $_{70.7}$ & 26 & 370 & .061 & $\stackrel{\mathrm{A}}{\mathrm{V}}$ & $\begin{array}{l}87 \\
82\end{array}$ & $\begin{array}{r}102 \\
98\end{array}$ & $\begin{array}{l}116 \\
109\end{array}$ & $\begin{array}{l}126 \\
117\end{array}$ & $\begin{array}{l}134 \\
123\end{array}$ & $\begin{array}{l}140 \\
127\end{array}$ & $\begin{array}{l}144 \\
130\end{array}$ & $\begin{array}{l}146 \\
132\end{array}$ & $\begin{array}{l}146 \\
133\end{array}$ & $\begin{array}{l}141 \\
133\end{array}$ & $\begin{array}{l}135 \\
132\end{array}$ & $\begin{array}{l}127 \\
125\end{array}$ & $\begin{array}{l}122 \\
117\end{array}$ & \\
\hline$\underset{69.7}{R_{6} G_{.}}$ & 36 & 360 & .082 & $\stackrel{\mathbf{A}}{\mathbf{V}}$ & $\begin{array}{l}89 \\
88\end{array}$ & $\begin{array}{l}109 \\
105\end{array}$ & $\begin{array}{l}120 \\
114\end{array}$ & $\begin{array}{l}127 \\
121\end{array}$ & $\begin{array}{l}131 \\
125\end{array}$ & $\begin{array}{l}133 \\
128\end{array}$ & $\begin{array}{l}134 \\
130\end{array}$ & $\begin{array}{l}135 \\
131\end{array}$ & $\begin{array}{l}135 \\
127\end{array}$ & $\begin{array}{l}122 \\
113\end{array}$ & $\begin{array}{l}109 \\
101\end{array}$ & $\begin{array}{l}97 \\
90\end{array}$ & $\begin{array}{l}87 \\
81\end{array}$ & \\
\hline $\mathrm{J}_{70.5} \mathrm{C}_{.}$ & 32 & 295 & .069 & $\stackrel{\mathrm{A}}{\mathrm{V}}$ & $\begin{array}{l}93 \\
91\end{array}$ & $\begin{array}{l}123 \\
113\end{array}$ & $\begin{array}{l}138 \\
126\end{array}$ & $\begin{array}{l}149 \\
136\end{array}$ & $\begin{array}{l}150 \\
138\end{array}$ & $\begin{array}{l}139 \\
113\end{array}$ & $\begin{array}{r}118 \\
92\end{array}$ & $\begin{array}{l}98 \\
80\end{array}$ & $\begin{array}{l}82 \\
71\end{array}$ & & & & & \\
\hline P. W. & 21 & 270 & .073 & $\stackrel{\mathrm{A}}{\mathrm{V}}$ & $\begin{array}{r}100 \\
95\end{array}$ & $\begin{array}{l}117 \\
109\end{array}$ & $\begin{array}{l}128 \\
118\end{array}$ & $\begin{array}{l}134 \\
123\end{array}$ & $\begin{array}{l}134 \\
122\end{array}$ & $\begin{array}{l}129 \\
111\end{array}$ & $\begin{array}{r}120 \\
94\end{array}$ & $\begin{array}{r}108 \\
85\end{array}$ & $\begin{array}{l}97 \\
80\end{array}$ & $\begin{array}{l}88 \\
75\end{array}$ & $\begin{array}{l}79 \\
71\end{array}$ & $\begin{array}{l}72 \\
68\end{array}$ & $\begin{array}{l}70 \\
66\end{array}$ & \\
\hline J. $H$. & 21 & 270 & .061 & $\stackrel{\mathrm{A}}{\mathrm{V}}$ & $\begin{array}{l}96 \\
92\end{array}$ & $\begin{array}{l}111 \\
105\end{array}$ & $\begin{array}{l}122 \\
115\end{array}$ & $\begin{array}{l}130 \\
122\end{array}$ & $\begin{array}{l}134 \\
125\end{array}$ & $\begin{array}{l}134 \\
122\end{array}$ & $\begin{array}{l}132 \\
110\end{array}$ & $\begin{array}{l}126 \\
101\end{array}$ & $\begin{array}{r}118 \\
92\end{array}$ & $\begin{array}{r}108 \\
84\end{array}$ & $\begin{array}{r}100 \\
76\end{array}$ & $\begin{array}{l}94 \\
69\end{array}$ & & \\
\hline$\underset{88}{\text { A. M. }}$ & 27 & 250 & .054 & $\stackrel{\mathbf{A}}{\mathbf{V}}$ & $\begin{array}{l}92 \\
83\end{array}$ & $\begin{array}{r}100 \\
92\end{array}$ & $\begin{array}{r}106 \\
98\end{array}$ & $\begin{array}{l}110 \\
102\end{array}$ & $\begin{array}{l}110 \\
103\end{array}$ & $\begin{array}{r}109 \\
99\end{array}$ & $\begin{array}{r}106 \\
94\end{array}$ & $\begin{array}{r}101 \\
88\end{array}$ & $\begin{array}{l}97 \\
83\end{array}$ & $\begin{array}{l}93 \\
78\end{array}$ & $\begin{array}{l}88 \\
74\end{array}$ & $\begin{array}{l}83 \\
69\end{array}$ & $\begin{array}{l}77 \\
65\end{array}$ & \\
\hline R.S. & 32 & 300 & .059 & $A$ & $\begin{array}{l}94 \\
93\end{array}$ & $\begin{array}{l}117 \\
110\end{array}$ & $\begin{array}{l}133 \\
124\end{array}$ & $\begin{array}{l}142 \\
134\end{array}$ & $\begin{array}{l}146 \\
139\end{array}$ & $\begin{array}{l}146 \\
139\end{array}$ & $\begin{array}{l}146 \\
139\end{array}$ & $\begin{array}{l}146 \\
139\end{array}$ & $\begin{array}{l}142 \\
136\end{array}$ & $\begin{array}{l}136 \\
130\end{array}$ & $\begin{array}{l}128 \\
123\end{array}$ & $\begin{array}{l}118 \\
112\end{array}$ & $\begin{array}{r}108 \\
98\end{array}$ & $\begin{array}{l}97 \\
82\end{array}$ \\
\hline$\underset{58.6}{\text { W. H. }}$ & 24 & 300 & .061 & A & $\begin{array}{l}85 \\
82\end{array}$ & $\begin{array}{l}112 \\
101\end{array}$ & $\begin{array}{l}128 \\
116\end{array}$ & $\begin{array}{l}137 \\
126\end{array}$ & $\begin{array}{l}142 \\
132\end{array}$ & $\begin{array}{l}141 \\
134\end{array}$ & $\begin{array}{l}137 \\
126\end{array}$ & $\begin{array}{l}129 \\
113\end{array}$ & $\begin{array}{l}119 \\
104\end{array}$ & $\begin{array}{r}108 \\
88\end{array}$ & $\begin{array}{l}95 \\
78\end{array}$ & $\begin{array}{l}84 \\
71\end{array}$ & $\begin{array}{l}76 \\
63\end{array}$ & \\
\hline K. W. & 38 & 260 & .069 & $\stackrel{\mathbf{A}}{\mathrm{V}}$ & $\begin{array}{l}84 \\
85\end{array}$ & $\begin{array}{l}105 \\
101\end{array}$ & $\begin{array}{l}113 \\
108\end{array}$ & $\begin{array}{l}118 \\
108\end{array}$ & $\begin{array}{l}122 \\
105\end{array}$ & $\begin{array}{r}125 \\
98\end{array}$ & $\begin{array}{r}124 \\
90\end{array}$ & $\begin{array}{r}121 \\
83\end{array}$ & $\begin{array}{r}115 \\
77\end{array}$ & $\begin{array}{r}108 \\
71\end{array}$ & $\begin{array}{r}102 \\
66\end{array}$ & $\begin{array}{l}97 \\
61\end{array}$ & $\begin{array}{l}92 \\
58\end{array}$ & $\begin{array}{l}88 \\
55\end{array}$ \\
\hline
\end{tabular}

* Blood sugar values at 10-minute intervals taken from curves constructed from determinations on samples (30 to 40 per subject) drawn at staggered time intervals which varied in the different subjects.

t A-Capillary blood sugar (mg. per cent). V-Antecubital vein sugar (mg. per cent).

$\uparrow$ Beginning of insulin infusion.

$A-V / A$ values ${ }^{8}$ with insulin were significantly increased over control values $(P=.01)$.

The data on the 12 subjects who received a combined infusion of insulin and glucagon after an initial control period are shown in Table II and one of the experiments is plotted in Figure 2. In eight subjects of this group there was a marked arterial hyperglycemia (over $200 \mathrm{mg}$. per cent) when glucagon was added to the glucose-insulin infusion mixture; in three there was little or no increase in arterial blood sugar over control values. In 11 of the 12 subjects a marked increase in $\mathrm{A}-\mathrm{V}$ glucose differences occurred (see Table II). The

$8 \mathrm{~A}-\mathrm{V} / \mathrm{A}=$ arterio-venous glucose difference divided by arterial glucose concentration. This value has been shown to be proportional to the specific rate constant of peripheral glucose utilization under the special conditions of the experiments (2). three subjects who showed little or no arterial hyperglycemia after the addition of glucagon were, nevertheless, among those who exhibited marked increases in $\mathrm{A}-\mathrm{V}$ glucose differences.

A comparison of the data of the insulin plus glucagon group with the groups who received glucagon (previously reported [2]) or insulin alone revealed the following noteworthy differences: (a) the A-V/A values for the insulin plus glucagon group were significantly greater $(P=.001)$ than those for either of the other two groups (Figure 3); (b) the arterial hyperglycemia of the insulin plus glucagon group was often greater than that of the glucagon group despite the fact that the mean glucose infusion rates ${ }^{4}$ of the former

4 The mean glucose infusion rates (mg. per minute) for the three groups were: glucagon 321 ; insulin 312 ; and insulin plus glucagon 296 . 
were slightly lower than those of the latter, and (c) the fall in blood sugar below initial fasting levels so commonly observed in the insulin group did not occur ${ }^{5}$ in the insulin plus glucagon group.

\section{DISCUSSION}

That insulin enhances the peripheral utilization of glucose is now widely accepted. The recent demonstration (2) that glucagon has a similar action which is independent of a secondary release of insulin has already been cited. The experiments reported here indicate that when glucagon and insulin are administered together peripheral glu-

S The only exceptions are the last two venous values on subject $\mathrm{T}$. $\mathrm{H}$. cose utilization is increased beyond that with either hormone alone. In addition, the opposing effect of the two on arterial blood sugar may result, at times, in a virtually unchanged level. The greater arterial hyperglycemia often observed with glucagon plus insulin as compared with glucagon alone may have resulted from higher liver glycogen levels induced by the insulin which was administered 15 to 25 minutes before the glucagon in the subjects who received both hormones.

These findings suggest that insulin and glucagon are capable of functioning jointly to enhance the peripheral utilization of glucose and at the same time, of counteracting the opposing effect of each other on the blood sugar. Judging from the variation in responses observed in different subjects

TABLE II

Insulin + glucagon cases*

\begin{tabular}{|c|c|c|c|c|c|c|c|c|c|c|c|c|c|c|c|c|c|c|c|}
\hline \multirow{2}{*}{$\begin{array}{c}\text { Patient } \\
\text { Wt. } \\
\left(K_{g .}\right)\end{array}$} & \multirow[b]{2}{*}{ Age } & \multirow{2}{*}{$\begin{array}{c}\text { Glucose } \\
\text { mg./. } \\
\text { min. }\end{array}$} & \multirow{2}{*}{$\begin{array}{c}\text { Insulin } \\
\text { units/ } \\
\text { min. }\end{array}$} & & \multicolumn{15}{|c|}{ Time in minutes } \\
\hline & & & & & o & 10 & 20 & 30 & 40 & 50 & 60 & 70 & 80 & 90 & 100 & 110 & 120 & 130 & 140 \\
\hline$\overline{71.8}$ & 33 & 370 & .086 & $\begin{array}{l}\text { Af } \\
\mathrm{V} \ddagger\end{array}$ & $\begin{array}{l}86 \\
83\end{array}$ & $\begin{array}{r}103 \\
98\end{array}$ & $\begin{array}{l}114 \\
109\end{array}$ & $\begin{array}{l}121 \\
116\end{array}$ & $\begin{array}{l}126 \\
121\end{array}$ & $\begin{array}{l}130 \\
124\end{array}$ & $\begin{array}{l}133 \\
127\end{array}$ & $\begin{array}{l}134 \\
127\end{array}$ & $\begin{array}{l}136 \\
128\end{array}$ & $\begin{array}{l}158 \\
141\end{array}$ & $\begin{array}{l}164 \\
137\end{array}$ & $\begin{array}{l}163 \\
133\end{array}$ & $\begin{array}{l}151 \\
129\end{array}$ & & \\
\hline D. D. & 39 & 316 & .111 & $\begin{array}{l}\mathbf{A} \\
\mathbf{V}\end{array}$ & & $\begin{array}{l}130 \\
124\end{array}$ & $\begin{array}{l}136 \\
129\end{array}$ & $\begin{array}{l}141 \\
133\end{array}$ & $\begin{array}{l}145 \\
136\end{array}$ & $\begin{array}{l}138 \\
126\end{array}$ & $\begin{array}{l}127 \\
106\end{array}$ & $\begin{array}{l}128 \\
105\end{array}$ & $\begin{array}{l}140 \\
110\end{array}$ & $\begin{array}{l}133 \\
110\end{array}$ & $\begin{array}{l}126 \\
105\end{array}$ & $\begin{array}{r}119 \\
99\end{array}$ & $\begin{array}{r}114 \\
96\end{array}$ & & \\
\hline$\underset{66.4}{\text { M. R. } \dagger}$ & 27 & 333 & .065 & $\begin{array}{l}\mathbf{A} \\
\mathbf{V}\end{array}$ & & $\begin{array}{l}149 \\
132\end{array}$ & $\begin{array}{l}150 \\
134\end{array}$ & $\begin{array}{l}153 \\
136\end{array}$ & $\left|\begin{array}{l}161 \\
141\end{array}\right|$ & $\begin{array}{l}162 \\
145\end{array}$ & $\begin{array}{l}175 \\
138\end{array}$ & $\begin{array}{l}200 \\
153\end{array}$ & $\begin{array}{l}212 \\
159\end{array}$ & $\begin{array}{l}209 \\
155\end{array}$ & $\begin{array}{l}189 \\
147\end{array}$ & $\begin{array}{l}174 \\
137\end{array}$ & $\begin{array}{l}165 \\
126\end{array}$ & & \\
\hline T. D. & 37 & 265 & .062 & $\begin{array}{l}\mathbf{A} \\
\mathbf{V}\end{array}$ & $\begin{array}{l}106 \\
100\end{array}$ & $\begin{array}{l}128 \\
118\end{array}$ & $\begin{array}{l}143 \\
132\end{array}$ & $\begin{array}{l}150 \\
140\end{array}$ & $\begin{array}{l}155 \\
145\end{array}$ & $\begin{array}{l}157 \\
140\end{array}$ & $\begin{array}{l}184 \\
142\end{array}$ & $\begin{array}{l}208 \\
145\end{array}$ & $\begin{array}{l}220 \\
142\end{array}$ & $\begin{array}{l}222 \\
138\end{array}$ & $\begin{array}{l}217 \\
136\end{array}$ & $\begin{array}{l}208 \\
138\end{array}$ & $\begin{array}{l}197 \\
145\end{array}$ & $\begin{array}{l}185 \\
138\end{array}$ & $\begin{array}{l}172 \\
110\end{array}$ \\
\hline J. G. & 26 & 275 & .084 & $\begin{array}{l}\mathbf{A} \\
\mathbf{V}\end{array}$ & $\begin{array}{r}100 \\
95\end{array}$ & $\begin{array}{l}108 \\
100\end{array}$ & $\begin{array}{l}121 \\
112\end{array}$ & $\begin{array}{l}130 \\
121\end{array}$ & $\begin{array}{l}135 \\
127\end{array}$ & $\begin{array}{l}140 \\
132\end{array}$ & $\begin{array}{l}163 \\
145\end{array}$ & $\begin{array}{l}189 \\
171\end{array}$ & $\begin{array}{l}201 \\
186\end{array}$ & $\begin{array}{l}197 \\
184\end{array}$ & $\begin{array}{l}183 \\
171\end{array}$ & $\begin{array}{l}167 \\
160\end{array}$ & $\begin{array}{l}157 \\
152\end{array}$ & $\begin{array}{l}149 \\
144\end{array}$ & $\begin{array}{l}134 \\
128\end{array}$ \\
\hline$\underset{73.4}{\text { E. }}$ & 37 & 270 & .058 & $\mathbf{A}$ & $\begin{array}{l}93 \\
91\end{array}$ & $\begin{array}{l}110 \\
104\end{array}$ & $\begin{array}{l}121 \\
113\end{array}$ & $\begin{array}{l}126 \\
118\end{array}$ & $\begin{array}{l}129 \\
120\end{array}$ & $\begin{array}{l}129 \\
120\end{array}$ & $\begin{array}{l}136 \\
123\end{array}$ & $\begin{array}{l}163 \\
141\end{array}$ & $\begin{array}{l}172 \\
143\end{array}$ & $\begin{array}{l}172 \\
137\end{array}$ & $\begin{array}{l}159 \\
128\end{array}$ & $\begin{array}{l}145 \\
119\end{array}$ & $\begin{array}{l}132 \\
110\end{array}$ & $\begin{array}{l}122 \\
103\end{array}$ & $\begin{array}{l}113 \\
100\end{array}$ \\
\hline$\underset{73.6}{\text { A. } 0 .}$ & 43 & 320 & .064 & $\begin{array}{l}\mathbf{A} \\
\mathbf{V}\end{array}$ & $\begin{array}{l}88 \\
85\end{array}$ & $\begin{array}{r}100 \\
96\end{array}$ & $\begin{array}{l}110 \\
105\end{array}$ & $\begin{array}{l}119 \\
114\end{array}$ & $\begin{array}{l}125 \\
119\end{array}$ & $\begin{array}{l}135 \\
124\end{array}$ & $\begin{array}{l}166 \\
136\end{array}$ & $\begin{array}{l}193 \\
155\end{array}$ & $\begin{array}{l}213 \\
167\end{array}$ & $\begin{array}{l}219 \\
174\end{array}$ & $\begin{array}{l}212 \\
171\end{array}$ & $\begin{array}{l}205 \\
154\end{array}$ & $\begin{array}{l}202 \\
148\end{array}$ & $\begin{array}{l}197 \\
153\end{array}$ & $\begin{array}{l}176 \\
147\end{array}$ \\
\hline C. $\mathrm{R}$. & 23 & 310 & .054 & $\begin{array}{l}\mathrm{A} \\
\mathrm{V}\end{array}$ & $\begin{array}{l}82 \\
82\end{array}$ & $\begin{array}{l}101 \\
101\end{array}$ & $\begin{array}{l}115 \\
109\end{array}$ & $\begin{array}{l}124 \\
113\end{array}$ & $\begin{array}{l}129 \\
114\end{array}$ & $\begin{array}{l}130 \\
115\end{array}$ & $\begin{array}{l}161 \\
110\end{array}$ & $\begin{array}{l}198 \\
136\end{array}$ & $\begin{array}{l}213 \\
140\end{array}$ & $\begin{array}{l}201 \\
137\end{array}$ & $\begin{array}{l}192 \\
122\end{array}$ & $\begin{array}{l}184 \\
116\end{array}$ & $\begin{array}{l}177 \\
121\end{array}$ & $\begin{array}{l}170 \\
120\end{array}$ & $\begin{array}{l}163 \\
116\end{array}$ \\
\hline R. $R$ R. & 29 & 290 & .062 & $\begin{array}{l}\mathbf{A} \\
\mathbf{V}\end{array}$ & $\begin{array}{l}95 \\
92\end{array}$ & $\begin{array}{l}113 \\
106\end{array}$ & $\begin{array}{l}121 \\
114\end{array}$ & $\begin{array}{l}127 \\
120\end{array}$ & $\mid \begin{array}{l}131 \\
124\end{array}$ & $\begin{array}{l}135 \\
128\end{array}$ & $\begin{array}{l}146 \\
116\end{array}$ & $\begin{array}{l}185 \\
163\end{array}$ & $\begin{array}{l}199 \\
187\end{array}$ & $\begin{array}{l}203 \\
197\end{array}$ & $\begin{array}{l}185 \\
181\end{array}$ & $\begin{array}{l}170 \\
167\end{array}$ & $\begin{array}{l}160 \\
156\end{array}$ & $\begin{array}{l}151 \\
147\end{array}$ & $\begin{array}{l}137 \\
134\end{array}$ \\
\hline $\mathrm{J.}_{80.5}$ & 34 & 290 & .061 & $\begin{array}{l}\mathbf{A} \\
\mathbf{V}\end{array}$ & $\begin{array}{l}90 \\
90\end{array}$ & $\begin{array}{l}108 \\
101\end{array}$ & $\begin{array}{l}121 \\
112\end{array}$ & $\begin{array}{l}129 \\
121\end{array}$ & $\mid \begin{array}{l}133 \\
127\end{array}$ & $\begin{array}{l}135 \\
121\end{array}$ & $\begin{array}{l}163 \\
133\end{array}$ & $\begin{array}{l}189 \\
145\end{array}$ & $\begin{array}{l}202 \\
150\end{array}$ & $\begin{array}{l}197 \\
151\end{array}$ & $\begin{array}{l}190 \\
150\end{array}$ & $\begin{array}{l}184 \\
149\end{array}$ & $\begin{array}{l}180 \\
147\end{array}$ & $\begin{array}{l}177 \\
147\end{array}$ & $\begin{array}{l}176 \\
151\end{array}$ \\
\hline$\underset{75.5}{\mathrm{~T} . \mathrm{H}}$ & 38 & 250 & .065 & $\stackrel{\mathrm{A}}{\mathrm{V}}$ & $\begin{array}{l}92 \\
92\end{array}$ & $\begin{array}{l}111 \\
104\end{array}$ & $\begin{array}{l}119 \\
112\end{array}$ & $\begin{array}{l}123 \\
117\end{array}$ & $\begin{array}{l}126 \\
119\end{array}$ & $\begin{array}{l}128 \\
121\end{array}$ & $\begin{array}{l}149 \\
122\end{array}$ & $\begin{array}{l}167 \\
136\end{array}$ & $\begin{array}{l}166 \\
140\end{array}$ & $\begin{array}{l}151 \\
123\end{array}$ & $\begin{array}{l}138 \\
113\end{array}$ & $\begin{array}{l}126 \\
105\end{array}$ & $\begin{array}{r}118 \\
97\end{array}$ & $\begin{array}{r}107 \\
85\end{array}$ & $\begin{array}{l}91 \\
71\end{array}$ \\
\hline J. & 38 & 300 & .077 & $\stackrel{\mathbf{A}}{\mathbf{V}}$ & $\begin{array}{l}98 \\
92\end{array}$ & $\begin{array}{l}122 \\
113\end{array}$ & $\begin{array}{l}141 \\
128\end{array}$ & $\begin{array}{l}154 \\
137\end{array}$ & $\mid \begin{array}{l}161 \\
141\end{array}$ & $\begin{array}{l}158 \\
141\end{array}$ & $\begin{array}{l}179 \\
154\end{array}$ & $\begin{array}{l}213 \\
170\end{array}$ & $\begin{array}{l}227 \\
174\end{array}$ & $\begin{array}{l}230 \\
173\end{array}$ & $\begin{array}{l}229 \\
173\end{array}$ & $\begin{array}{l}224 \\
180\end{array}$ & $\begin{array}{l}212 \\
184\end{array}$ & $\begin{array}{l}199 \\
185\end{array}$ & $\begin{array}{r}198 \\
-183\end{array}$ \\
\hline
\end{tabular}

* Blood sugar values at 10 -minute intervals taken from curves constructed from determinations on samples ( 30 to 40 per subject) drawn at staggered time intervals which varied in the different subjects.

Control period data not used for statistical analysis because a loading dose of glucose was used.

₹-A-Capillary blood sugar (mg. per cent). V-Antecubital vein sugar (mg. per cent).

$\uparrow$ Beginning of insulin infusion. $\uparrow \uparrow$ Beginning of glucagon infusion. 


\section{THE EFFECT OF INSULIN ON BLOOD GLUCOSE}

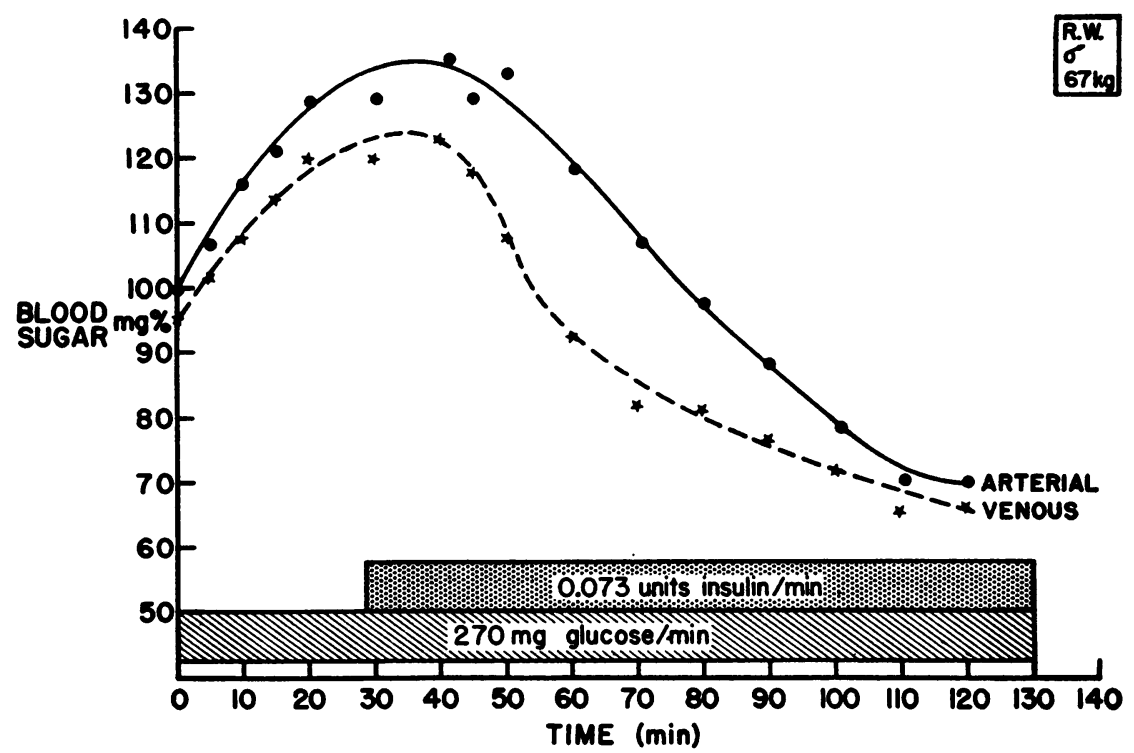

Fig. 1. The Effect of a Continuous Intravenous Infusion of Insulin on Arterial and Venous Blood Sugar Levels in a Normal MaN

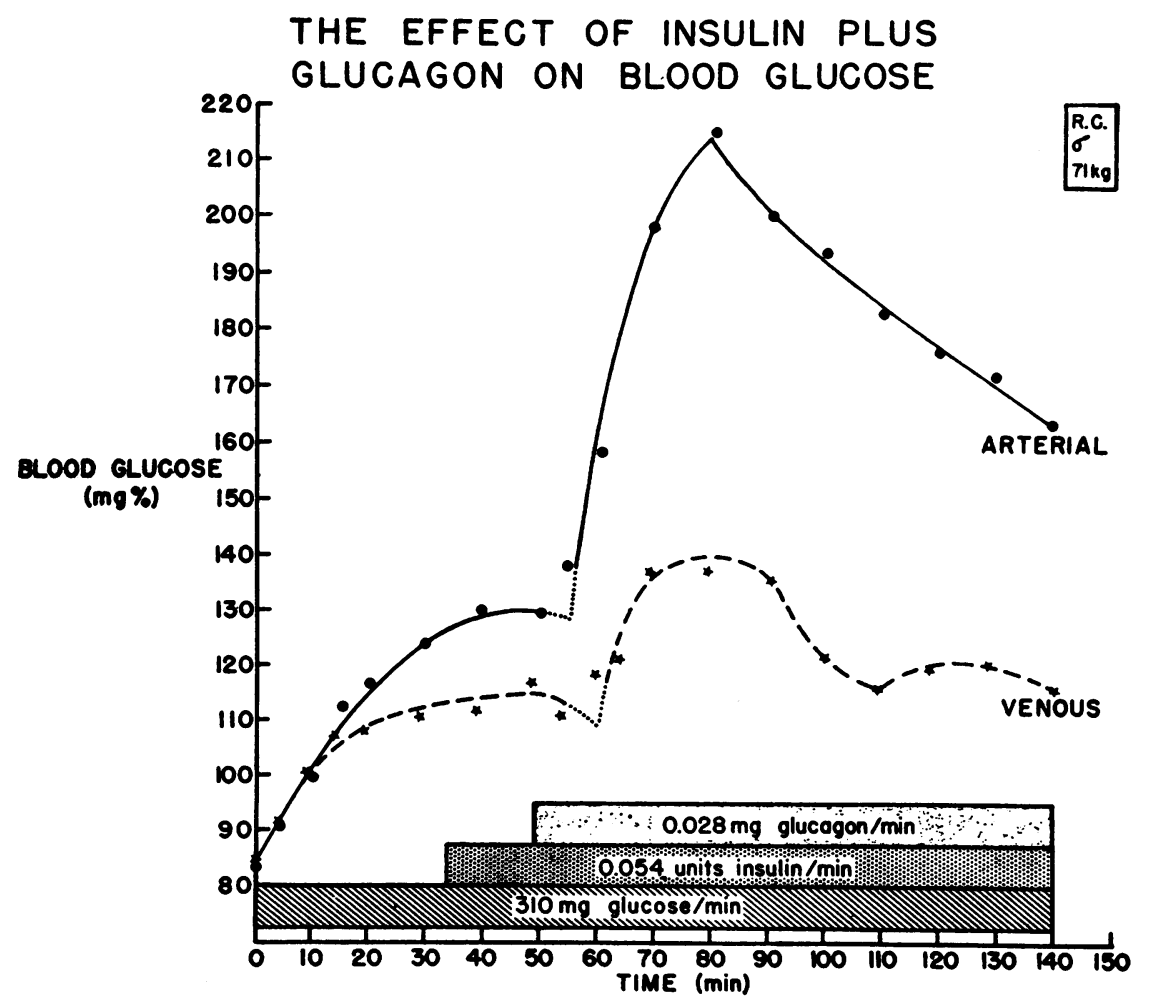

Fig. 2. The Effect of a Continuous Intravenous Infusion of Glucagon Plus Insulin on Arterial and Venous Blood Sugar Levels in a Normal Man 


\section{THE EFFECT OF GLUCAGON AND INSULIN ON PERIPHERAL UTILIZATION OF GLUCOSE}

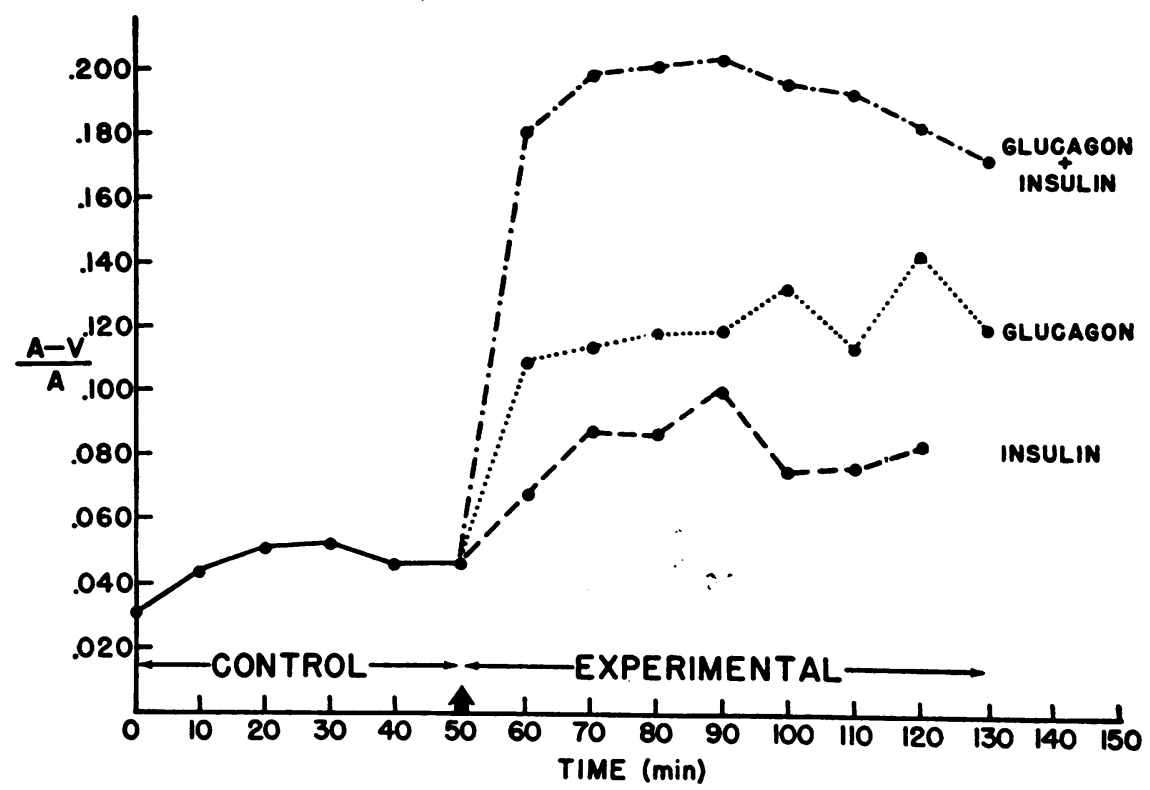

Fig. 3. A Summary of Continuous Intravenous Infusion Experiments in Normal Man Showing the Difference Between the Effect of Insulin, Glucagon, and Glucagon Plus Insulin on an Index of the Peripheral Utilization of Glucose (A-V/A)

Each point of the control period represents the median value of 54 subjects, whereas each point of the three experimental groups represents the median value of 12 subjects. The data on the glucagon subjects were taken from a previous publication (2).

receiving similar doses, there is considerable individual difference in responsiveness to the two hormones. No correlation of response with body weight was observed.

The fact that glucagon and insulin have opposing actions on blood glucose and liver glycogen levels has led many to postulate that they are antagonists $(8,9)$. Another concept of glucagon's role has been advanced by Bürger (10) and by Pincus and Rutman (11). These workers suggest that glucagon acts as an adjunct to insulin because it mobilizes liver glycogen for the enhancing action of insulin on glucose utilization in the peripheral tissues.

It has been reported that glucagon inhibits the action of insulin on glucose uptake by the isolated rat diaphragm (12). This was a preliminary report on work done before purified preparations of glucagon were available. Smith in F. G. Young's laboratory was unable to confirm these results (13). Ingle, Nezamis, and Humphrey observed no effect of glucagon on the disposal of administered glucose in the eviscerated rat (14). Drury, Wick, and Sherill reported that in the eviscerated rabbit glucagon had no effect on glucose oxidation but had a slight inhibiting effect on the disappearance of blood glucose (15). The apparent discrepancy between some of these findings and those reported here might be explained by the studies of Lang, Goldstein, and Levine (16). These workers have presented evidence suggesting that the liver contains a "humoral agent" which enhances peripheral glucose utilization even in the presence of maximal insulin dosage. It is conceivable that glucagon causes the release of this liver factor and that this accounts for the difference between the results in the normal human as compared with the eviscerated animal and the isolated rat diaphragm. In keeping with this hypothesis is the observation that glucagon has no effect on blood sugar levels or arterio-venous glucose differences in the hepatectomized dog (17). 


\section{SUMMARY}

Studies on the effect of glucagon and insulin on the disappearance of blood glucose in 56 normal men using a constant glucose infusion technique are reported. The results indicate that the two hormones administered together (a) cause a significantly greater increase in peripheral glucose utilization than either one alone, and (b) may result in a neutralization of the opposing action of each other on arterial blood sugar levels. These findings suggest that glucagon and insulin may function jointly to regulate the level of blood glucose and the utilization of carbohydrate by peripheral tissues.

\section{ACKNOWLEDGMENTS}

We are indebted to Dr. Lewis Bernstein for assistance with the statistical analysis, to Dr. Thomas A. Witten for constant interest and helpful advice, and to Dr. Cosmo G. Mackenzie for advice on the preparation of the manuscript.

\section{REFERENCES}

1. Elrick, H., Discussion on influence of growth hormone on human metabolism in International Symposium on The Hypophyseal Growth Hormone, Nature and Actions. New York, McGraw-Hill, 1955, pp. 561-568.

2. Elrick, H., Hlad, C. J., Jr., and Witten, T., The enhancement of peripheral glucose utilization by glucagon. J. Clin. Invest., 1955, 34, 1830.

3. Goss, C. M., Gray's Anatomy of the Human Body. 26th ed., Philadelphia, Lea Febiger, 1954, p. 737.

4. Schaeffer, J. P., Morris' Human Anatomy. 11th ed., New York, The Blakiston Co., 1953, p. 774.

5. Somogyi, M., Studies of arteriovenous differences in blood sugar. I. Effect of alimentary hyperglycemia on the rate of extrahepatic glucose assimilation. J. Biol. Chem., 1948, 174, 189.

6. Nelson, N., A photometric adaptation of the Somogyi method for the determination of glucose. J. Biol. Chem., 1944, 153, 375.

7. Mood, A. M., Introduction to the Theory of Statistics. New York, McGraw-Hill Book Co., Inc., 1950, pp. 385-418.

8. DeDuve, C., Glucagon, the hyperglycemic glycogenolytic factor of the pancreas. Lancet, 1953, 2, 99.

9. Foa, P. P., Glucagon, the hyperglycemic-glycogenolytic hormone of the pancreas. Advances Int. Med., 1954, 6, 29.

10 Bürger, M., Das Glucagon. Fortschr. d. Diagnostik u. Therapie, 1950, 1, 225.

11. Pincus, I. J., and Rutman, J. Z., Glucagon, the hyperglycemic agent in pancreatic extracts. A possible factor in certain types of diabetes. Arch. Int. Med., 1953, 92, 666.

12. R.-Candela, J. L., Inhibitory effect of pancreas extract and H-G factor on the insulin glucose uptake of the isolated diaphragm. Ciba Foundation Colloquia on Endocrinology. Vol. VI, Boston, Little, Brown, 1953, pp. 233-241.

13. Young, F. G., Discussion of above reference (13) Ciba Foundation Colloquia on Endocrinology. Vol. VI, Boston, Little, Brown, 1953, pp. 241-242.

14. Ingle, D. J., Nezamis, J. E., and Humphrey, L. M., Absence of hyperglycemic effect of glucagon in the eviscerate rat. Proc. Soc. Exper. Biol. \& Med., 1953, 84, 232.

15. Drury, D. R., Wick, A. N., and Sherill, J. W., The effect of the hyperglycemic factor on the metabolism of glucose by the extrahepatic tissues. Diabetes, 1954, 3, 129.

16. Lang, S., Goldstein, M. S., and Levine, R., Influence of the liver on uptake of glucose by extrahepatic tissues. Am. J. Physiol., 1954, 177, 447.

17. Elrick, H., Glucagon and the regulation of carbohydrate metabolism. Nature, 1956, In press.

\section{SPECIAL NOTICE TO SUBSCRIBERS}

Post Offices will no longer forward the Journal when you move.

Please notify The Journal of Clinical Investigation, Business Office, 622 West 168th Street, New York 32, N. Y. at once when you have a change of address, and do not omit the zone number if there is one. 